\title{
Las carteras de alianzas en el sector español de la construcción: análisis de las redes ego
}

\author{
Cristóbal Casanueva Rocha \\ Ignacio Castro Abancéns ${ }^{1}$ \\ Jose Luis Galán González
}

Universidad de Sevilla. Departamento de Administración de empresas y marketing

\begin{abstract}
Resumen
Este trabajo trata de avanzar en el estudio de las carteras de alianzas estratégicas aprovechando la potencialidad que ofrece el análisis de las redes sociales (ARS). En el estudio de las carteras de alianzas existen tres cuestiones principales de investigación que son: la emergencia, la configuración y la gestión de la cartera. Esta investigación se centra en la configuración de la cartera de alianzas, es decir, en el contenido y la estructura de la red egocéntrica del actor focal. Con este objetivo fueron analizadas 10.556 uniones temporales de empresas (UTE) realizadas por 229 empresas que desarrollan su actividad en el sector español de la construcción de obra pública. Los resultados muestran que las empresas analizadas siguen distintos comportamientos estratégicos a la hora de configurar sus carteras de alianzas.
\end{abstract}

Palabras clave: cartera de alianzas - redes egocéntricas - configuración de cartera - grupos estratégicos - bloques estructurales.

\begin{abstract}
This research tries to deepen in the analysis of alliance portfolio leveraging the potential of social network analysis (SNA). There are three research questions in this analysis: the emergence, the configuration and the managing of an alliance portfolio. This work focuses on the configuration of alliance portfolio, that is, on the content and the structure of the focal firm's egocentric network. With this aim 10.556 strategic alliances (UTE) established by 229 firms from public works industry were analyzed. The results show that the firms undertake different strategic behavior patterns in the configuration of its alliance portfolios.
\end{abstract}

Key words: Alliance portfolio - egocentric networks -configuration of alliance portfolio- strategic groups -structural blockmodel.

\footnotetext{
1 Enviar correspondencia a icastro@us.es. Facultad de Económicas de la Universidad de Sevilla. Departamento de Administración de Empresas y Marketing. Avenida Ramón y Cajal 1, 41018 Sevilla (España), Teléfono: 954554432, Fax: 954556989.
} 


\section{Introducción}

En las últimas décadas las empresas han recurrido ampliamente a las alianzas estratégicas para competir más adecuadamente o crecer. De esta forma, muchas empresas se encuentran incrustadas en una densa red de relaciones interorganizativas con competidores, clientes, proveedores y otras organizaciones (Hoffmann 2007). En consecuencia, el estudio de las alianzas entre empresas se ha convertido en un tópico relevante de investigación en el campo del estudio de las organizaciones. Recientemente, la atención se ha desplazado desde el nivel de la alianza individual al estudio de la cartera de alianzas que una empresa ha generado (Sarkar, Aulakh \& Madhok 2009). Según los estudios de redes, una cartera de alianzas se puede entender como la red egocéntrica de las alianzas de una empresa, es decir, la cartera o portfolio de alianzas estaría conformada por todos los vínculos directos con las empresas socios (Baum, Calabrese \& Silverman 2000, Ozcan, Eisenhardt 2009). En una revisión reciente sobre el tema, Wassmer (2010) señaló la existencia de tres cuestiones principales de investigación, cuyo desarrollo se encuentra aún incipiente: la emergencia, la configuración y la gestión de la cartera de alianzas.

El presente estudio aborda algunos de los aspectos de la segunda cuestión señalada por Wassmer (2010), la configuración de la cartera de alianzas, es decir, el contenido y estructura de la red de alianzas en la que una empresa se halla inmersa, para lo cual se aplicará el Análisis de Redes Sociales (ARS). Desde un punto de vista operativo, se trata de analizar la egonet de una empresa en el conjunto de sus relaciones interorganizativas con el objetivo de estudiar cómo la empresa va configurando su cartera de alianzas de manera estratégica. Los estudios anteriores no han aprovechado la potencialidad del Análisis de Redes Sociales para estudiar estas redes ego. Este trabajo propone analizar la cartera de alianzas de las empresas a partir del estudio de las características de sus redes egocéntricas, de los factores atributivos de las empresas y de la diferencias entre empresas por su pertenencia a diferentes grupos estratégicos. Se trata de un estudio fundamentalmente exploratorio, dada la ausencia de investigaciones que hayan abordado esta cuestión con una muestra de empresas y de relaciones de cooperación suficientemente amplia.

El contexto de estudio está constituido por las uniones temporales de empresas (UTES) que se han desarrollado en la obra pública en España en el periodo 19992010 de las 229 empresas de la construcción que más cooperan. En total son más de 10.000 las alianzas analizadas en el periodo. 
Utilizando el ARS se estudian cuatro cuestiones de investigación o aspectos de esta realidad empresarial. En primer lugar, las características de las redes egocéntricas de las empresas, relacionándolas con diversos atributos de las empresas. En segundo lugar, se analiza si las empresas tienen comportamientos homofílicos, es decir, si se relacionan con otras empresas que juegan el mismo papel o rol en la red o no. En tercer lugar, se tratará de identificar conglomerados o clúster jerárquicos a partir de indicadores relacionales, así como determinar bloques relacionales a partir de la idea de la equivalencia estructural; es decir bloques de empresas que siguen similares patrones relacionales. Finalmente, en cuarto lugar, se analizará si los grupos estratégicos identificados a partir de una serie de dimensiones se relacionan con los bloques relacionales.

\section{Contexto de análisis: el sector español de la obra pública}

\section{La construcción en España}

El sector español de la construcción juega un papel relevante tanto en el ámbito nacional como internacional. En España la construcción es una actividad claramente determinante respecto a su crecimiento económico; así, en términos de Valor Añadido Bruto (VAB) representó en el 2008 más del 10,9 por 100 del PIB, del 13,9 por 100 del empleo y del 58,7 por 100 de la inversión, además de generar potentes efectos indirectos inducidos de arrastre, siendo precisamente este sector el que más ha contribuido al crecimiento del Producto Interior Bruto de España (PIB), sólo superado en algunas regiones por el sector servicios (SEOPAN 2006). Concretamente, la construcción en los últimos años explica más del el 21,5 por 100 del crecimiento del PIB de España y el 43,8 por 100 del incremento del empleo tiene su origen en el ciclo de la construcción. Respecto a la producción de la construcción, en el año 2008 se cifró en 204.982 millones de euros. De esta producción, la obra civil representó el $24 \%$ de la producción total, y el $76 \%$ restante de la producción correspondió a la edificación (residencial, no residencial y rehabilitación).

\section{Companías españolas de la construcción}

La industria española de la construcción se caracteriza por ser un sector relativamente fragmentado con una oferta bastante heterogénea en donde las medianas y grandes empresas representan un porcentaje muy reducido del total del sector $(4 \%)$. Sin embargo, son estas empresas las que juegan un papel 
fundamental en la licitación de obra pública, aunque luego recurran a la subcontratación en la ejecución de las mismas. En España existen 6 grandes grupos constructores que dominan el ámbito nacional y también el internacional. Estos grupos son: ACS (Dragados), Ferrovial, FCC, Sacyr, Acciona y OHL.

Siguiendo los criterios de la Comisión Europea, dentro del sector de la construcción es posible identificar cuatro subsectores: obra civil, edificación residencial, edificación no residencial y rehabilitación y mantenimiento de edificios. El mercado de edificación residencial se caracteriza porque los demandantes son esencialmente promotores, inmobiliarias y cooperativas de vivienda, que subcontratan la construcción de la vivienda sobre unos terrenos de los que son propietarios. Este subsector representa un $34.5 \%$ del la construcción total, y sus clientes fundamentalmente son privados $y$, en un porcentaje mucho menor, público. El subsector de la edificación no residencial abarca la construcción de oficinas, fábricas, almacenes, naves industriales, naves comerciales, etc., así como edificios destinados a usos sanitarios, docentes, militares, administrativos o de uso institucional. En este mercado, por tanto, concurren clientes públicos y privados, y representó el $16.2 \%$. El mercado de rehabilitación y mantenimiento de edificios, tanto residenciales como no residenciales, representó el $24 \%$ del sector de la construcción. Por último, el mercado de obra civil engloba un gran número de actividades relacionadas con la construcción de infraestructuras viales, ferroviarias, marítimas, hidráulicas, etc., así como su mantenimiento. En el año 2007 alcanzó un $24.5 \%$ del sector de la construcción experimentando un crecimiento del $9 \%$. Desde el punto de vista de la oferta, las constructoras para poder concurrir a una determinada licitación deben poseer la clasificación administrativa requerida, disponer de la necesaria capacidad técnica y ser acreedor de solvencia económica y financiera. Esto hace que a los proyectos de cuantía reducida acudan un gran número de empresas $\mathrm{y}$, sin embargo, a los grandes proyectos de obra civil concurran un número reducido de grandes constructoras y otro muy elevado de medianas y pequeñas empresas que subcontratan con aquéllas o concurren en las licitaciones asociadas entre sí, generalmente en una coalición de proyecto o consorcio. Este subsector se caracteriza por el hecho de que los grandes grupos constructores ejecutan alrededor de un $30 \%$ de la obra civil y el restante $70 \%$ es realizado por empresas que poseen cuotas de mercado en torno al $1 \%$. Ninguna constructora individualmente controla más del $10 \%$ del mercado y las principales constructoras poseen una cuota bastante similar. Por tanto, en relación con la obra pública se podría afirmar que existe un grado medio de concentración aunque éste es reducido si se compara con otros países de su entorno (Ministerio de Economía 
de España 2003). Además, el número de licitadores respecto a las licitaciones de proyectos de cuantías considerables se está reduciendo como consecuencia del constante proceso de concentración que está experimentado el sector de la construcción, donde están surgiendo grandes holdings diversificados, y de la proliferación de proyectos conjuntos o en coalición.

Por último, en relación con la oferta del sector de la construcción, es importante resaltar que en este sector la separación entre las empresas es más ambigua o difusa que en otros sectores, pues en la mayor parte de los proyectos terminan cooperando o trabajando de manera conjunta empresas de diferentes dimensiones, bien a través de las Uniones Temporales de Empresas (UTE), o bien mediante la subcontratación. En este sentido, podríamos decir que la industria de la construcción, considerada de manera global como una sola, se encuentra bastante fragmentada. Pero, profundizando en el sector, observamos que esto no es así. En primer lugar, existen numerosas empresas que de manera repetida trabajan conjuntamente (proyecto conjunto (UTE) y subcontratación). En segundo lugar, si consideramos la industria de la construcción como un conglomerado de mercados, no todas las empresas de arquitectura son capaces de diseñar hospitales y colegios, al igual que no todas las empresas de ingeniería son capaces de diseñar puentes, carreteras, obras hidráulicas, torres, etc. Lo mismo ocurre con las constructoras y los subcontratistas: hay grupos de empresas que compiten unas con otras, pero todas no compiten contra todas. Es decir, las empresas normalmente se focalizan en un mercado en lugar de competir con cada una de las empresas del sector en todos los mercados; aunque lógicamente algunas de ellas desarrollan su actividad en más de un mercado. Así, el presente trabajo se va a centrar en las empresas que trabajan en el mercado de la obra pública, donde las principales constructoras poseen una cuota de mercado en torno al $40 \%$.

Desde el punto de vista de la demanda, el mercado de la obra civil se caracteriza porque los demandantes son generalmente Administraciones Públicas, de ahí que se identifique obra civil con obra pública. Debido a las características de los demandantes, tanto el grado de concentración como el poder de negociación de los mismos son muy elevados. En este mercado de obra pública en España existe una normativa específica que regula todo el proceso de contratación que exige la organización de licitaciones para adjudicar los proyectos con el fin de proporcionar transparencia en la formación de precios. En el año 2009 la licitación pública en España alcanzó los 39.100,40 millones de euros $(€)$. 
Uno de los elementos clave en los últimos años del buen funcionamiento de la actividad de la construcción en España ha sido, sin lugar a dudas, la obra pública, que ha mostrado una tendencia constantemente creciente. En este sentido, la crisis inmobiliaria que en estos años (2007/08) castiga a las economías de los países más desarrollado esta haciendo que países como España estén apostando por la obra pública tanto en la edificación como en obra civil con el objetivo de amortiguar la crisis en el sector global de la construcción en términos de empleo y PIB.

\section{La red de las empresas de la construcción en españa}

Para analizar cómo se configura la estructura de la cartera de alianzas en el sector de la obra pública en España se ha elaborado una red a partir de la conformación de Uniones Temporales de Empresas (UTE) que se han producido en el sector entre los años 1999 y 2010. Como se ha comentado anteriormente, en el sector de la construcción los proyectos son generalmente realizados conjuntamente por varias empresas, principalmente, a través de dos mecanismos: las alianzas estratégicas o coaliciones y la subcontratación. Eccles (1981), en un estudio internacional sobre el sector de la construcción, analiza las relaciones entre los constructores generales y los subcontratistas, identificando que estos vínculos son generalmente estables y continuos a través de largos períodos de tiempo, y estas relaciones son raramente establecidas a través de pujas competitivas. Este investigador considera que este fenómeno constituye una "quasi-integración", que genera como resultado una "quasifirma" (Eccles 1981).

En la investigación que se propone sería tarea bastante difícil controlar toda la información acerca de la subcontratación que se produce en la red de constructores. Por esta razón, se ha decidido elegir como relación fundamental para construir la red de construcción los proyectos conjuntos o en coalición, que en España adoptan una forma concreta denominada Uniones Temporales de Empresas (UTE), que se hayan presentado a las licitaciones y se hayan adjudicado algún proyecto de obra pública durante el periodo analizado. Al ubicar la investigación en obra pública, esta información debe reflejarse por ley en los boletines oficiales.

La UTE es un sistema de colaboración entre empresarios, sociedades o no, por tiempo cierto, determinado o indeterminado, para el desarrollo o ejecución de una obra, servicio o suministro. Una Unión Temporal de Empresas implica, por tanto, una actividad de colaboración entre empresas durante un periodo de tiempo en el cual las empresas intercambian recursos, información, y desarrollan habilidades conjuntas (Ahuja 2000a, Koka, Prescott 2002). Las organizaciones que conforman 
una UTE podrán ser personas físicas o jurídicas con residencia en España o en el extranjero. La duración máxima será de diez años, prorrogable de año en año, para aquellas Uniones Temporales de Empresas que se hallen inscritas en el Registro Especial del Ministerio de Hacienda, siendo esta inscripción de carácter voluntario para las empresas. Las UTEs carecen de personalidad jurídica propia; por esta razón, la responsabilidad frente a terceros recae de manera solidaria e ilimitada en las empresas que conforman la alianza. Sin lugar a dudas, esta forma de colaboración es especialmente frecuente en el sector de la construcción gozando de un trato privilegiado en materia fiscal.

Concluyendo, las uniones temporales de empresas o acuerdos de colaboración facilitan la competitividad entre grandes empresas y empresas de tamaño medio o pequeño, y emergen como un componente significativo dentro de la estrategia de las constructoras para alcanzar y mantener una ventaja competitiva; las empresas adoptan estos acuerdos de colaboración como respuesta ante la dinámica de cambios producidos en el entorno (Madhavan, Koka, \& Prescott, 1998).

Para obtener la muestra de empresas del sector de la construcción que permitiese desarrollar el análisis se utilizó una base de datos (Maninvest.com) que recogía todas la adjudicaciones publicadas en los boletines oficiales: DOCE, BOE, Autonómicos (17 Boletines, además de Ceuta y Melilla), y Provinciales (43 boletines) desde enero de 1999 a diciembre de 2010. Partiendo de estos datos se plantearon dos criterios de selección:

a) Criterio relacional: la población de estudio está conformada por aquellas empresas que hayan participado al menos en dos Uniones Temporales de Empresas (UTE). En este criterio se emplea una perspectiva realista, ya que las uniones temporales de empresas son entidades sociales reales, que surgen con la motivación o finalidad de alcanzar la adjudicación del proyecto al que licitan y son perfectamente delimitadas y definidas por los actores, desde el mismo momento en que su existencia se plasma en un contrato pública. Respecto al hecho de establecer como criterio dos UTE, algunos investigadores sugieren que los límites de la red analizada pueden ser diseñados sobre la base de la frecuencia de la interacción, requiriéndose no sólo que exista una interacción sino que además lo haga con una determinada frecuencia (Laumann, Marsden, \& Prensky, 1983).

b) Criterio atributivo: en el cual se seguirá una perspectiva nominalista al generar una selección de empresas en función de una serie de características atributivas o individuales de las mismas. La primera característica es que aparezcan recogidos sus datos individuales en la base de datos DUNS 50.000 o en su defecto 
en DICODI 50.000, lo cual conlleva otros sub-criterios de selección como son: un determinado volumen de facturación, o que sean personas jurídicas y no físicas. La segunda característica o atributo que deberán cumplir las empresas para ser incluidas en la muestra consiste en pertenecer a la red de constructores que compite en el mercado de la obra pública. Para aplicar este criterio se va a emplear como regla general el código SIC.

Aplicando estos criterios se obtuvo una muestra de 229 empresas, las cuales tenían los códigos SIC 15, 16 ó 67 en primer lugar y, por tanto, pertenecían a la red de constructores, y que, además, habían llevado a cabo al menos dos UTEs en el período considerado. En este sentido, en el periodo analizado que va desde enero de 1999 a diciembre de 2010, las administraciones públicas han licitado en obras públicos un importe de 375.987,64 millones de euros y las 229 que conforman nuestra muestra se han adjudicado el $88 \%$ de ese importe, por consiguiente, podemos afirmar que el grado de representatividad de la muestra seleccionada es muy elevado.

En este artículo se han analizado 10.556 alianzas entre esas empresas supusieron un importe total de 93.410 millones de euros frente a los 100.944 millones de euros de adjudicaciones de manera individual, lo que supone que prácticamente la mitad de las adjudicaciones o del negocio de las constructoras se produce a través de alianzas o UTES.

La red se ha conformado a través de dos referencias básicas: el número de UTES que han realizado dos empresas de la muestra y el importe global de esas alianzas conjuntas durante el periodo considerado. Esto ha dado lugar a tres matrices de relaciones diferenciadas. La primera de las matrices que se ha considerado y que hemos llamado VÍNCULOS, se refiere a la existencia o no de alguna alianza entre las 229 empresas que conforman la muestra. Es decir, en la matriz aparece un 1 en la casilla si las dos empresas representadas en la fila y la columna que convergen en esa celda han tenido al menos una UTE en el periodo y un 0 en caso contrario. La segunda de las matrices, denominada NÚMERO UTES, se refiere al número de alianzas que dos empresas cualesquiera han mantenido durante el periodo. Es decir, el valor de una celda representará el número de UTEs entre cada par de empresas. La tercera matriz, Ilamada IMPORTE UTES, recoge el importe total de las adjudicaciones en las que han participado dos empresas durante el periodo estudiado.

En el Gráfico 1 aparecen recogidos los vínculos entre las empresas de la obra pública en España en el periodo 1999-2010. En el mismo se puede observar una 
estructura en forma de estrella con una parte central con mucha densidad, una periferia con muy pocos vínculos y un pequeño número de elementos aislados. Sin embargo, para estudiar de una forma más analítica una red es preciso sobrepasar el análisis gráfico y conocer los principales indicadores de red que proporciona el Análisis de Redes Sociales (Wasserman, Faust 1994, Hanneman, R. A. and Riddle, M. 2005) y que aparecen en la Tabla 1. En dicha tabla aparecen recogidos los indicadores de la matriz VÍNCULOS, ya que los correspondientes a las otras dos matrices son iguales en su mayor parte o difíciles de interpretar en otros de ellos, debido a que se ha considerado la intensidad de los vínculos entre las empresas. La matriz Vínculos es la forma dicotomizada de las otras dos matrices que suele ser la base de cálculo de las principales medidas de la estructura de las redes.

La Tabla 1 muestra que el tamaño de la red es de 229 empresas, como había sido comentado anteriormente, y que entre ellas la densidad en la realización de las UTEs es de 0.0317. La densidad es el número de vínculos reales en relación del número de vínculos posibles. Los datos de densidad de esta red señalan que se dan algo más de un $3 \%$ de todas las relaciones posibles entre las empresas. La estructura de esa red muestra un pequeño número de elementos aislados (17). La existencia de elementos aislados se debe a que son empresas que han participado en UTEs durante el periodo, pero no lo han hecho con otras empresas de la muestra. El resto de las empresas están conectadas entre sí en el componente principal de la misma, que está conformado por 212 empresas que mantienen vínculos directos o indirectos entre sí, lo que supone el $90.98 \%$ de las empresas de la muestra. Además existen dos componentes más con sólo dos elementos cada uno. Por tanto, se trata de una red muy cohesionada. Las medidas de centralización de la red (Freeman 1978) también muestran un fenómeno parecido. La centralización de grado es la extensión a nivel de la red de la centralidad de grado, esto es, el número de socios con los que una empresa focal ha mantenido alianzas. La centralización de grado es una medida que indica el porcentaje en que la red se parece a una estrella. En este caso es de un $45.49 \%$, que es una cifra muy alta e indica que se trata de una red bastante centralizada. También presenta un valor alto (34.63\% la centralización de intermediación). La intermediación se refiere a las posibilidades de que las empresas de la red de alianzas aprovechen su posición de intermediarios o brokers para conectar a otras partes o elementos de la red por los caminos más cortos. Esta alta centralización de intermediación indica que hay actores que pueden aprovechar dicha posición estratégica en la red. La última medida considerada es el coeficiente Clustering, que representa la propensión de la red a formar vínculos alrededor de grupos muy cohesionados (es la media de las 
densidades de las redes egocéntricas de todas las empresas de la red). En la red analizada el coeficiente es elevado, 0.394, por lo que la densidad de la cartera de alianzas es también importante. Es decir, en promedio existen un número elevado de vínculos entre los socios que conforman la cartera de alianzas de la empresa focal.

Las redes también pueden ser analizadas en su estructura si se consideran las posiciones más relevantes que ocupan las empresas que conforman la misma. Sin entrar en un análisis detallado de las posiciones de la red, la Tabla 2 recoge los valores de las 10 empresas más centrales en las tres redes que han sido estudiadas. Se analizan dos formas de centralidad: la centralidad de grado (referida al número y la fuerza de los vínculos de cada empresa con las otras de la red) y la centralidad de intermediación (referida a cómo una empresa puede aprovechar su posición porque muchos de los caminos más cortos entre otras dos empresas de la red pasen por ella para conectarse). La centralidad de intermediación no tiene en cuenta el peso o la intensidad de los vínculos, por lo que los valores son los mismos para las tres redes. En cambio, la centralidad de grado sí los tiene en cuenta, así que los valores son diferentes y las empresas que conforman el ranking de las diez primeras variarán según la magnitud estudiada.

La Tabla 2 muestra que la posición más central desde todos los parámetros utilizados la ocupa la empresa Dragados, por lo que parece que la red pivota claramente alrededor de un punto central. En realidad, es más bien una zona central, ya que se puede apreciar que en las cuatro medidas usadas (tres de centralidad de grado y la de centralidad de intermediación) las empresas que conforman el ranking de las diez primeras no varían mucho, aunque sí sus posiciones relativas. En general, se trata de los más importantes grupos de construcción y obra pública españoles y algunos no tan grandes en tamaño pero que muestran una alta actividad en UTEs.

Precisamente el Gráfico 2 intenta mostrar esa estructura de la red presentando el grafo de la red egocéntrica de la empresa Dragados. En dicho grafo se está representando básicamente el centro de la red global de alianzas y las mayores relaciones de Dragados se producen con las otras grandes empresas del sector. El grosor de las líneas refleja la intensidad relativa de las relaciones de cooperación en función del número de alianzas que han mantenido las empresas. 
Tabla 1. Indicadores de redes para la relación Vínculos.

\begin{tabular}{|l|l|}
\hline Tamaño de la red & 229 \\
\hline Densidad & 0.0317 \\
\hline Número de componentes & 20 \\
\hline Número de aislados & 17 \\
\hline Tamaño del componente principal & 212 \\
\hline Porcentaje del componente principal & $90.98 \%$ \\
\hline Centralización de grado & $45.49 \%$ \\
\hline Centralización de intermediación & $34.63 \%$ \\
\hline Clustering Coefficient & 0.394 \\
\hline
\end{tabular}

Tabla 2. Indicadores de las empresas principales

\begin{tabular}{|c|c|c|c|c|c|c|c|}
\hline \multicolumn{2}{|c|}{ GRADO VÍNCULOS } & \multicolumn{2}{|c|}{ GRADO NOUTES } & \multicolumn{2}{|c|}{ GRADO IMPORTE UTE } & \multicolumn{2}{|c|}{ INTERMEDIACIÓN } \\
\hline DRAGAD & 112 & DRAGAD & 709 & DRAGAD & $\begin{array}{l}1104520806 \\
0\end{array}$ & DRAGAD & $\begin{array}{l}9402.8837 \\
9\end{array}$ \\
\hline FCC & 60 & FCC & 629 & API & 8806316032 & FERROVIAL & $\begin{array}{l}2877.8737 \\
8\end{array}$ \\
\hline FERROVIAL & 55 & FERROVIAL & 511 & COPASA & 4178773760 & FCC & $\begin{array}{l}2661.0476 \\
1\end{array}$ \\
\hline $\mathrm{OHL}$ & 42 & $\mathrm{OHL}$ & 356 & ACSA & 3510356224 & $\mathrm{OHL}$ & $\begin{array}{l}1280.1763 \\
9\end{array}$ \\
\hline COMSA & 35 & COPISA & 298 & SACYR & 3115620352 & VIASCON & $\begin{array}{l}1175.3538 \\
8\end{array}$ \\
\hline ACCION & 34 & COPCISA & 264 & FCC & 3102654720 & SACYR & 1026.1936 \\
\hline SACYR & 32 & ACCION & 228 & $\mathrm{OHL}$ & 2308490240 & ACSA & $\begin{array}{l}894.41302 \\
5\end{array}$ \\
\hline VIASCON & 32 & COMSA & 220 & CAVOSA & 2179070208 & INIESTA & $\begin{array}{l}830.52429 \\
2\end{array}$ \\
\hline AZVI & 31 & SACYR & 206 & COPCISA & 1732528640 & ISOLUX & $\begin{array}{l}743.59094 \\
2\end{array}$ \\
\hline COPCISA & 29 & DRACE & 159 & COPISA & 1715643520 & TEJER & $\begin{array}{l}643.03192 \\
1\end{array}$ \\
\hline
\end{tabular}


Gráfico 1. Grafo de las alianzas (UTE) entre empresas de obra pública 1999-2010

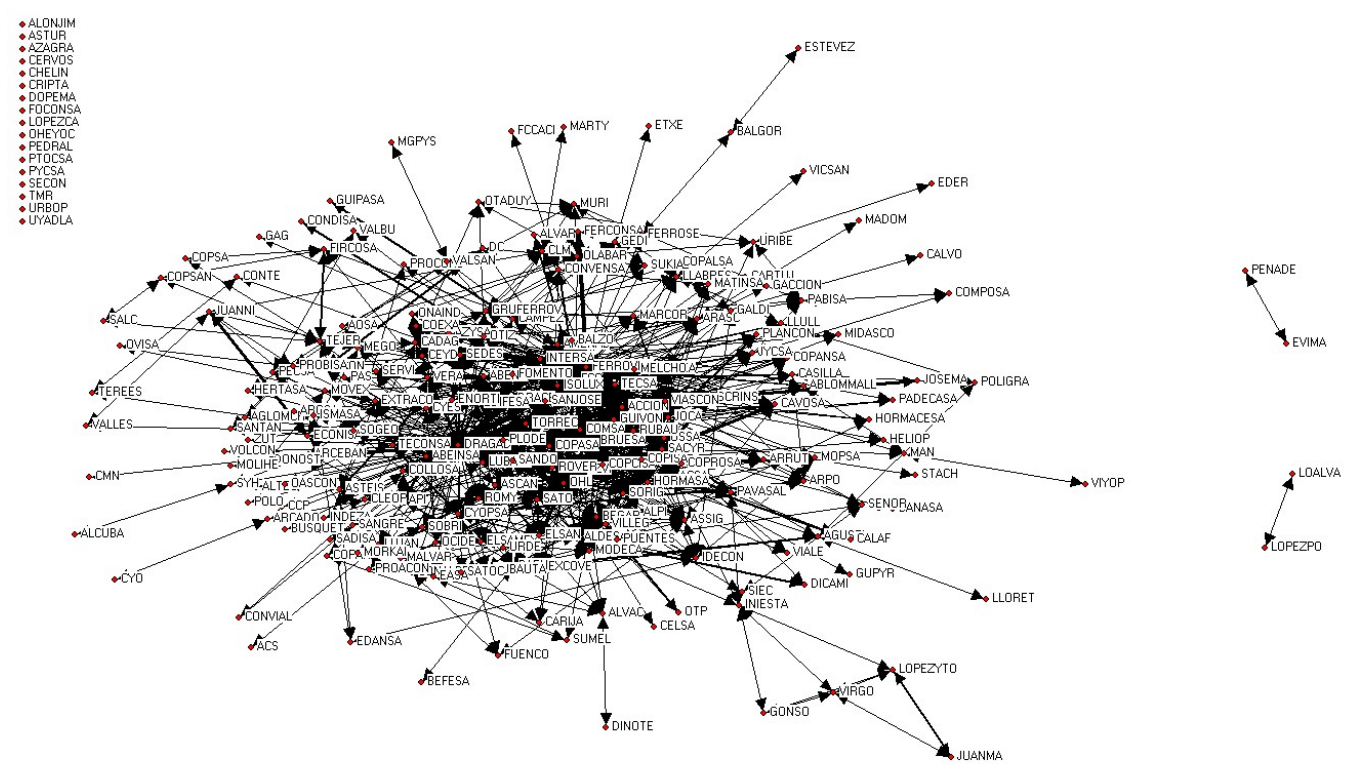

Gráfico 2. Grafo de la red egocéntrica de la empresa Dragados.

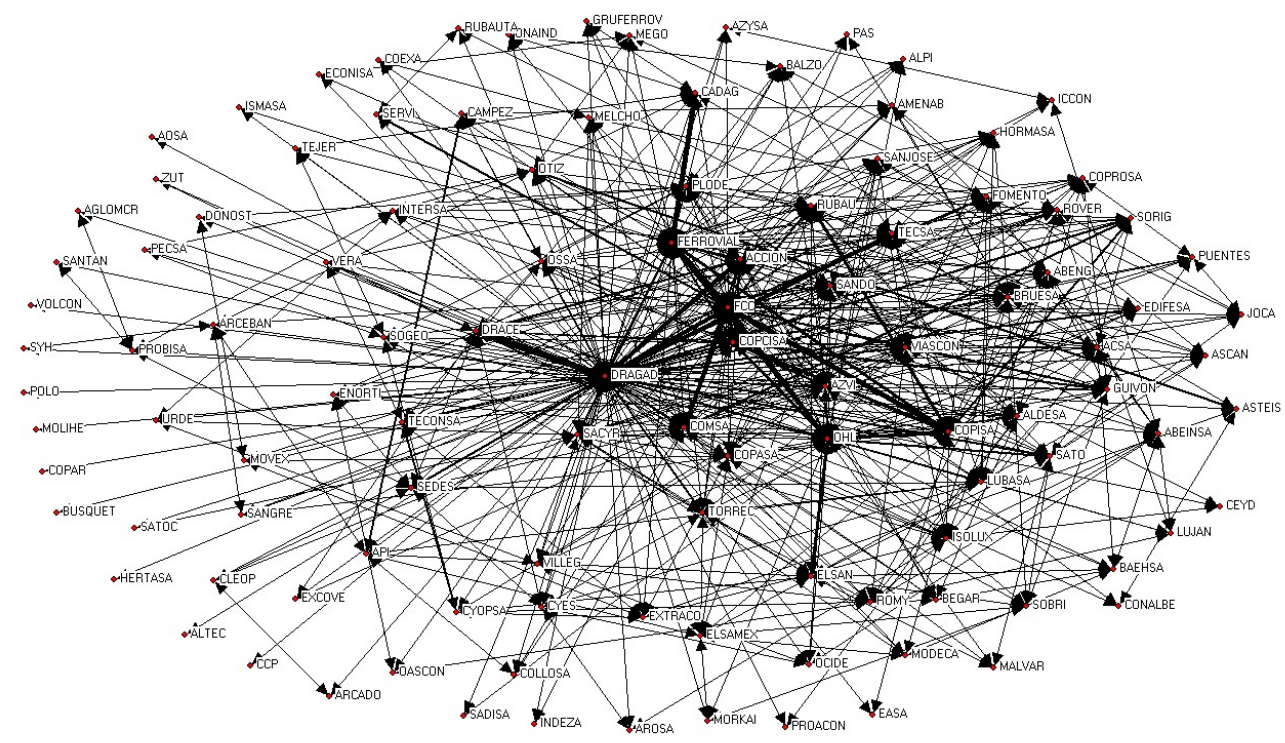

\section{Características de los portfolio de alianzas o redes ego}

En primer lugar, con el objetivo de conocer algunas características de las carteras de alianzas o redes ego de las distintas empresas que conforman nuestra muestra se planteó un análisis de correlaciones donde se analizaron dos tipos de indicadores, relacionales y atributivos: 
Indicadores relacionales los cuales se obtuvieron con el programa UCINET VI (Borgatti, Everett \& Freeman 2002). Limitación (CONSTRAINT), fue utilizada para operacionalizar el concepto de huecos estructurales de Burt (1992); así, un elevado nivel de limitación indica que los compañeros de la empresa están densamente conectados unos a otros $y$, por tanto, la redundancia en los flujos de información es muy elevada; mientras que un bajo valor de limitación indica que la empresa posee una red que está escasamente conectada (Koka, Prescott 2002, Hanneman, R. A. and Riddle, M. 2005, Burt 1992, Koka, Prescott 2008). El otro indicador desarrollado por Burt (1992) y que sigue una lógica similar es la jerarquía (JERARQ), que trata de evaluar si los actores que conforman la red mantienen o no una fuerte relación respecto a un actor central. En este sentido, en la medida en que los actores que conformen la red ego se encuentren relacionados de manera fuerte a un contacto central (jerarquía), la red estará densamente cohesionado y, por tanto existirán pocos huecos estructurales. El tamaño (SIZE) hace referencia al número de empresas que conforman la red ego o cartera de alianzas del actor focal. Tamaño efectivo (EffSize). Respecto al indicador denominado tamaño efectivo (Burt 1992), éste trata de medir el número de actores, ponderados por la fuerza de la relación, a los que se encuentra unido directamente el actor focal, menos un factor redundante Así, en el caso de que ninguno de los actores que conforman la red egocéntrica estuviesen conectados entre ellos, el tamaño efectivo coincidiría con el tamaño total de la red egocéntrica (SIZE). De esta manera, el tamaño efectivo representaría el $100 \%$ de eficiencia (Efficiency) (Borgatti 1997). El número de componentes débiles (nWeakComponents) hace referencia al número de bloques de empresas que quedarían desconectados en el caso de que quitásemos al actor ego de la red.

Indicadores atributivos de las empresas. El tamaño del ego o actor focal: medido tanto a nivel de número de empleados como de la facturación del último ejercicio. La actividad relacional: que fue medida comparando los ingresos procedentes de las obras realizadas por el actor ego en alianza con otras con la facturación total de la empresa. El ámbito de la empresa: hace referencia al porcentaje de las obras realizadas por la empresa que han sido adjudicadas por la administración central con el objetivo de diferenciarlas de las obras que tienen un carácter regional o local. 
Tabla 3. Tabla de correlaciones de indicadores atributivos y relacionales.

\begin{tabular}{|l|c|c|c|c|c|}
\hline & Size & nWeakComp & EffSize & Constraint & Hierarchy \\
\hline TAMAÑO EMPLEADOS &, $854^{* *}$ &, $409^{* *}$ &, $870^{* *}$ &,$- 247^{* *}$ &,- 106 \\
\hline TAMAÑO VENTA &, $839^{* *}$ &, $341^{* *}$ &, $852^{* *}$ &,$- 221^{* *}$ &,- 081 \\
\hline ACTIVIDAD RELACIONAL &, $226^{* *}$ &, 123 &, $182^{* *}$ &,$- 149^{*}$ &,$- 190^{* *}$ \\
\hline ÁMBITO NACIONAL &, $241^{* *}$ &, 031 &, $207^{* *}$ &,$- 286^{* *}$ &,$- 205^{* *}$ \\
\hline
\end{tabular}

\footnotetext{
**. La correlación es significativa al nivel 0,01 (bilateral).

*. La correlación es significante al nivel 0,05 (bilateral).
}

La tabla anterior (tabla 3) nos permite alcanzar las siguientes conclusiones. En primer lugar el tamaño de las carteras o portfolios de alianzas mantiene una importante correlación positiva con todos los indicadores atributivos. Es decir, las empresas que tienen una mayor dimensión, tanto en número de empleados como en facturación, son la que poseen una cartera de alianzas o portfolio de mayor dimensión. Esto parece lógico, ya que generalmente las UTES o consorcios se suelen establecer para la realización de proyectos u obras públicas importantes y son las empresas mayores las que disponen de superiores recursos y una mayor capacidad para asumir riesgos. Estas grandes empresas se asocian con otras por diversas razones: políticas, geográficas 0 por la especialización 0 complementariedad de recursos.

Otra conclusión que se puede deducir de la tabla 3 es que las empresas que tienen una actividad relacional grande, es decir, que una parte importante de su facturación procede de la realización de obras de manera conjunta con otras empresas, generalmente en UTEs, son las que disponen de las carteras de alianzas de mayor dimensión en comparación con aquellas otras empresas que tiene un comportamiento más individual y prefieren acudir a las licitaciones en solitario.

Una tercera conclusión hace referencia a que son aquellas empresas cuya ámbito de actuación es mayor, es decir, que acuden a las obras licitadas por la Administración Central para realizar una obra en cualquier parte del país, las que disponen de un portfolio de mayor tamaño, en comparación con aquellas empresas que tienen un ámbito más local o regional.

En cuarto lugar, podemos afirmar que las empresas que tienen un mayor tamaño, tienen redes egocéntricas o carteras con más tamaño efectivo o mayor eficiencia. 
Esto junto a la existencia de un mayor número de componentes débiles en la red egocéntrica proporciona al actor focal 0 ego mayores posibilidades de intermediación y tener que gestionar menores redundancias. Esto lógicamente va estar relacionado también con el tamaño de la red ego analizado anteriormente. Esto implica que cuanto mayor sean éstas, mayores serán las posibilidades de que existan huecos estructurales o posiciones de intermediación.

Por último, las empresas que tienen un ámbito de actuación nacional y con mayor actividad relacional son las que poseen unas carteras con mayor número de huecos estructurales. Estas redes egocéntricas van a tener unos niveles de jerarquía y limitación menores y, por tanto, mayores posibilidades de intermediación.

En definitiva, se puede afirmar que la estructura relacional de las carteras de alianzas está vinculada con el tamaño de la empresa y, en menor medida, con la intensidad de la actividad relacional de las firmas y con su ámbito de actuación.

\section{Análisis Clúster con indicadores relacionales}

Con el objetivo de profundizar en el comportamiento relacional de las empresas que conforman la muestra, procedimos a realizar con el software SPSS un análisis de conglomerados jerárquico a partir de los principales indicadores relacionales de las carteras de alianza o redes ego: tamaño de la cartera, tamaño efectivo de la red ego, jerarquía y limitación. A partir de la aplicación de este análisis se identificaron 4 grupos que se exponen a continuación. Como se puede apreciar, estamos trabajando con una muestra muy heterogénea donde un número reducido de empresas, entre 20 y 40 empresas, tiene una actividad relacional muy intensa y una dimensión internacional $y$, por otro lado, un importante número de empresas que intervienen de manera puntual o coyuntural con las grandes empresas. En consecuencia, dentro de un mismo sector y actividad productiva, las empresas diseñan diferentes carteras de alianzas, al menos desde un punto de vista relacional, y esas diferencias parecen depender en gran medida del tamaño de las compañías. 
Tabla 4. Análisis clúster de las redes ego.

\begin{tabular}{|l|l|}
\hline Clúster redes ego & \\
\hline Grupo 4 & FERROVIAL Y FCC \\
\hline Grupo 3 & $\begin{array}{l}\text { COMSA, COPCISA, COPISA, RUBAU, } \\
\text { BRUESA, VIASCON, SANDO, COPASA, } \\
\text { AZVI, OHL, TECSA, SACYR, ACCIONA E } \\
\text { ISOLUX }\end{array}$ \\
\hline Grupo 2 & $\begin{array}{l}\text { RESTO DE EMPRESAS QUE CONFORMAN } \\
\text { LA MUESTRA }\end{array}$ \\
\hline Grupo 1 & \\
\hline
\end{tabular}

Para tratar de entender mejor las estrategias relacionales de los 4 grupos identificados anteriormente, se procedió a realizar una serie de estadísticos descriptivos para cada unos de los conglomerados, que son mostrados en la tabla $6^{2}$.

2 Aunque nuestra muestra esta conformada por 229 empresas, al haber 17 elementos que están aislados en la red, a la hora de realizar los conglomerados con indicadores relacionales sólo ha tenido en cuenta 212 empresas. 
Tabla 5. Indicadores descriptivos de los clúster.

\begin{tabular}{|c|c|c|c|c|c|c|}
\hline \multicolumn{2}{|c|}{ Clusters } & Size & EffSize & Efficiency & Constraint & Hierarchy \\
\hline \multirow{5}{*}{1} & $\mathrm{~N}$ & 195 & 195 & 195 & 195 & 195 \\
\hline & Media & 5,50 & 3,895 & ,753 & ,414 & , 192 \\
\hline & Mínimo & 1 & ,999 &, 250 & ,0891 &,- 000 \\
\hline & Máximo & 18 & 12,888 & 1,000 & 1,000 & 1,000 \\
\hline & Desv. típ. & 4,333 & 3,059 & , 1968 & ,3126 & ,3792 \\
\hline \multirow{5}{*}{2} & $\mathrm{~N}$ & 14 & 14 & 14 & 14 & 14 \\
\hline & Media & 28,21 & 21,082 & ,739 & ,0720 & ,0427 \\
\hline & Mínimo & 20 & 14,100 & ,651 &, 0514 &, 0245 \\
\hline & Máximo & 42 & 33,761 & ,8593 & ,0880 & ,0819 \\
\hline & Desv. típ. & 6,351 & 5,946 & ,0582 & ,0112 & ,0170 \\
\hline \multirow{5}{*}{3} & $\mathrm{~N}$ & 1 & 1 & 1 & 1 & 1 \\
\hline & Media & 112,00 & 104,142 & ,929 & ,0276 & ,0774 \\
\hline & Mínimo & 112 & 104,142 & ,9298 & ,0276 & ,0774 \\
\hline & Máximo & 112 & 104,142 & ,929 & ,0276 & ,0774 \\
\hline & Desv. típ. & & . & . & . & . \\
\hline \multirow{5}{*}{4} & $\mathrm{~N}$ & 2 & 2 & 2 & 2 & 2 \\
\hline & Media & 57,50 & 49,559 & ,8620 & ,0448 & ,0749 \\
\hline & Mínimo & 55 & 47,618 & ,8583 & ,0442 & ,0742 \\
\hline & Máximo & 60 & 51,500 & ,8657 &, 0454 & ,0757 \\
\hline & Desv. típ. & 3,536 & 2,7448 & ,0052 & ,0008 & ,0011 \\
\hline \multirow{5}{*}{ Total } & $\mathrm{N}$ & 212 & 212 & 212 & 212 & 212 \\
\hline & Media & 7,99 & 5,934 & ,7542 & ,3864 &, 1805 \\
\hline & Mínimo & 1 & ,999 &, 250 & ,0276 &,- 0000 \\
\hline & Máximo & 112 & 104,142 & 1,000 & 1,000 & 1,000 \\
\hline & Desv. típ. & 11,278 & 9,676 & , 189 & ,3144 & ,3657 \\
\hline
\end{tabular}

En primer lugar, el grupo 3 esta conformado por una única empresa, Dragados, que surgió de la fusión de dos grandes grupos de la construcción como son ACS y Dragados. Este grupo posee de manera destacada el mayor portfolio de la muestra, muy por encima de la media del resto de grupos, y su red ego presenta una eficiencia del $92 \%$ presentando escasa redundancia y grandes posibilidades de intermediación.

Como veremos, en el siguiente apartado Dragados ocupa el centro de la red. En este sentido, es la empresa con más UTEs (990), superando incluso a su actividad de manera individual (891). En segundo lugar, el grupo 4 esta conformado por las empresas FCC y Ferrovial, las cuales son detrás de Dragados las empresas más activas a nivel relacional con 572 y 466 alianzas respectivamente. No obstante 
estas empresas, realizan más obras de manera individual (1098 y 760 respectivamente) que en alianzas. El Grupo 2 estaría integrado por el resto de las grandes empresas españolas, es decir, OHL, Acciona y Sacyr, así como, por las empresas grandes pero que tienen un ámbito nacional e incluso internacional como son Comsa, Copcisa, Copisa, Rubau, Bruesa, Viascon, Sando, Copasa, Azvi, Tecsa, e Isolux. Estas empresas poseen carteras de alianzas más pequeñas y cohesionadas en comparación con las del grupo 3 y 4 . Por último, el grupo 1 estaría conformado por un importante número de empresas de menor dimensión y ámbito de actuación.

\section{Análisis de las relaciones dentro de los grupos}

Para estudiar los patrones de las elecciones de los socios dependiendo de los distintos clústeres en los que hemos dividido la muestra objeto de análisis, se ha procedido a realizar "test de autocorrelaciones para una variable categórica"; es decir, se ha comprobado si las relaciones entre las empresas recogidas en las matrices de Vínculos, número de UTE e importe de UTE estaba ligada o no a la pertenencia a un determinado grupo estratégico ${ }^{3}$, definido en un vector como variable categórica. Ucinet realiza este procedimiento a través de un test de permutaciones que permite comprobar la significación del modelo y de las variables contenidas en el mismo.

Se han utilizado dos tipos de modelos recogidos en Ucinet. El primero es el Ilamado Variable Homofily, que intenta testar si para cada grupo, en nuestro caso para cada clúster, las empresas eligen relacionarse más con las de su propio clúster que con las de otros. Los resultados de este análisis aparecen recogidos en la tabla 6, para los tres grupos de datos que estamos manejando. El segundo es el llamado Structural Blockmodel. Este modelo plantea si las elecciones de cada grupo respecto a todos los demás es significativa o no. Los resultados aparecen en la tabla 7. Sin embargo, en los outputs de estos modelos aparecen dos hechos extraños: el primero de ellos es que no aparece las elecciones del último grupo consigo mismo y el segundo es que los coeficientes de elecciones entre grupos no son simétricos, cuando los datos sí lo son.

\footnotetext{
${ }^{3}$ La construcción de los grupos estratégicos se explica en el apartado 6.
} 
Tabla 6. Modelos de autocorrelación de redes con atributos categóricos. Variable homofilia.

\begin{tabular}{|l|l|l|l|l|l|l|}
\hline & \multicolumn{2}{l}{ VÍNCULOS } & \multicolumn{2}{l|}{ NÚMERO UTES } & \multicolumn{2}{l|}{ IMPORTE UTES } \\
\hline & Coef Stand & Significa & Coef Stand & Significa & Coef Stand & Significa \\
\hline Grupo 1 & -0.152977 & 0.000 & -0.073433 & 0.000 & -0.029246 & 0.000 \\
\hline Grupo 2 & 0.032858 & 0.008 & -0.003186 & 0.430 & -0.000198 & 0.726 \\
\hline Grupo 3 & 0.057515 & 0.000 & 0.039201 & 0.008 & 0.006004 & 0.019 \\
\hline Grupo 4 & 0.133121 & 0.000 & 0.309053 & 0.000 & 0.011874 & 0.005 \\
\hline R2 & $\mathbf{0 . 0 5 0}$ & & $\mathbf{0 . 1 0 4}$ & & $\mathbf{0 . 1 7 5}$ & \\
\hline $\begin{array}{l}\text { Sig } \\
\text { modelo }\end{array}$ & $\mathbf{0 . 0 0 0}$ & & $\mathbf{0 . 0 0 0}$ & & $\mathbf{0 . 0 0 0}$ & \\
\hline
\end{tabular}

Tabla 7. Modelos de autocorrelación de redes con atributos categóricos. Structural Blockmodel.

\begin{tabular}{|c|c|c|c|c|c|c|}
\hline & \multicolumn{2}{|c|}{ vínCULOS } & \multicolumn{2}{|c|}{ NÚMERO UTES } & \multicolumn{2}{|c|}{ IMPORTE UTES } \\
\hline & $\begin{array}{l}\text { Coef } \\
\text { Stand }\end{array}$ & Significa & Coef Stand & Significa & $\begin{array}{l}\text { Coef } \\
\text { Stand }\end{array}$ & Significa \\
\hline $1-2$ & 0.025268 & 0.082 & 0.006118 & 0.303 & 0.003149 & 0.219 \\
\hline $1-3$ & 0.044784 & 0.023 & 0.014788 & 0.138 & 0.004059 & 0.126 \\
\hline $1-4$ & 0.125857 & 0.000 & 0.052949 & 0.002 & 0.027432 & 0.001 \\
\hline $2-1$ & 0.025268 & 0.082 & 0.006118 & 0.303 & 0.003149 & 0.219 \\
\hline $2-2$ & 0.067989 & 0.000 & 0.013678 & 0.096 & 0.006519 & 0.050 \\
\hline $2-3$ & 0.069385 & 0.000 & 0.024976 & 0.011 & 0.023053 & 0.009 \\
\hline $2-4$ & 0.119934 & 0.000 & 0.061231 & 0.000 & 0.033160 & 0.007 \\
\hline $3-1$ & 0.044784 & 0.023 & 0.014788 & 0.138 & 0.004091 & 0.125 \\
\hline $3-2$ & 0.069385 & 0.000 & 0.024976 & 0.011 & 0.023053 & 0.009 \\
\hline $3-3$ & 0.072041 & 0.000 & 0.046173 & 0.006 & 0.008781 & 0.015 \\
\hline $3-4$ & 0.103875 & 0.000 & 0.177487 & 0.000 & 0.008833 & 0.012 \\
\hline $4-1$ & 0.125857 & 0.000 & 0.052949 & 0.002 & 0.027472 & 0.001 \\
\hline $4-2$ & 0.119934 & 0.000 & 0.061231 & 0.000 & 0.033160 & 0.007 \\
\hline $4-3$ & 0.103875 & 0.000 & 0.177487 & 0.000 & 0.008833 & 0.012 \\
\hline $4-4$ & 0.142102 & 0.000 & 0.313364 & 0.000 & 0.013591 & 0.005 \\
\hline$R 2$ & 0.114 & & 0.175 & & 0.201 & \\
\hline $\begin{array}{l}\text { Sig } \\
\text { modelo }\end{array}$ & 0.000 & & 0.000 & & 0.000 & \\
\hline
\end{tabular}


Los resultados que se muestran en la Tabla 6 señalan que los tres modelos analizados son significativos y que explican progresivamente una mayor proporción de la varianza, aunque en escasa cuantía lo cual nos permite concluir que en esta red los comportamientos homofílicos no son relevantes. No obstante, los datos presentan un patrón bien definido en las elecciones homofílicas del grupo 4 y 3 (las empresas más grandes), que prefieren aliarse con otras empresas de su propio grupo en ambos casos. También es constante la conducta del grupo 1 que prefiere en los tres grupos de datos (alianza o no con otra empresa, número de UTEs e importe de las UTEs) que prefiere aliarse con empresas de otros grupos antes que con las del suyo propio. Finalmente, las empresas del Grupo 2 presentan unos resultados diferentes para los tres grupos de datos. Si no se considera el peso de las relaciones, sino simplemente su existencia o no, parece que prefieren relacionarse con las de su propio grupo (aunque el nivel de significación es menor que el de los otros grupos). En caso de tener en cuenta la fuerza de las relaciones, aunque encontramos unos coeficientes negativos no podemos afirmar nada ya que las relaciones no son significativas.

La Tabla 7 muestra los resultados para los modelos que consideran los patrones de las elecciones entre cada par de grupos estratégicos. Los tres modelos (para cada una de las matrices) son significativos. En el caso de que no se tengan en cuenta los vínculos, la tabla muestra que todos los grupos quieren cooperar entre sí mismos y con los otros, salvo en dos casos. El primero de ellos se refiere a la cooperación entre los miembros del grupo 1, el más numeroso y el de las empresas más pequeñas. No aparecen los resultados en el modelo ${ }^{4}$, pero si atendemos a los resultados anteriores tendría un coeficiente con signo negativo y significativo, por lo que no quieren aliarse entre ellos. El segundo caso se refiere a los vínculos entre los grupos 1 y 2 cuyos resultados no son significativos. Si se considera como la fuerza de los vínculos entre las empresa el número de UTES que han realizado conjuntamente en el periodo analizado, tenemos algunos cambios en los grupos 1 y 2. Sobre las empresas del grupo 2 sólo podemos decir que quieren aliarse con las de los grupos 3 y 4 . Y sobre las del grupo 1 que se van a aliar con las del grupo 4 .

Finalmente si la fuerza de los vínculos se mide por el importe de las UTES que cada par de empresas han trabajado conjuntamente en el periodo estudiado, podemos observar que se producen las mismas pautas de relaciones que las que se daban

\footnotetext{
${ }^{4}$ Este análisis de varianza utiliza variables dummy y por esta razón una de las variables debe quedar fuera del análisis.
} 
cuando se consideraba como fuerza del vínculo el número de UTES, es decir, las del grupo 2 se alían con las de 3 y 4 y las del 1 sólo con las del 4.

\section{Grupos Estratégicos versus Bloques Relacionales}

\section{Grupos estratégicos}

Al igual que otros investigadores (Walker, Kogut \& Shan 1997, Nohria, Garcia-Pont 1991), a continuación trataremos de analizar de manera conjunta los bloques relacionales y los grupos estratégicos.

En primer lugar se procedió a construir los grupos estratégicos estableciendo tres dimensiones estratégicas, cada una de las cuales estará conformada, a su vez, por una serie de indicadores. Con estas tres dimensiones se van a tratar de identificar, mediante la herramienta estadística de los clúster o conglomerados jerárquicos, cuáles son los grupos más importantes o poderosos de la red.

- Indicadores individuales de la obra pública en España. Esta dimensión trata de captar la reputación que tiene la empresa en el mercado de la obra pública individualmente, sin cooperar o aliarse con ninguna empresa. Dentro de esta dimensión, los indicadores principales que se utilizaron fueron los siguientes. Un indicador era el ratio del importe de las adjudicaciones que la empresa había obtenido en su principal comunidad autónoma, consideración que se otorgaba a aquella comunidad donde la empresa se hubiese adjudicado un mayor importe de obras, dividido por el importe total de adjudicaciones que había obtenido la empresa a nivel individual en el periodo analizado (19992010). Si este ratio era pequeño, se podía considerar a la empresa de ámbito nacional y, si era muy grande, se consideraría a la empresa de ámbito local o regional. Otro indicador se calculaba como el cociente entre el importe de adjudicaciones que había obtenido la empresa en proyectos que superaban los 6 millones de euros dividido por el importe total que la empresa se hubiese adjudicado en el periodo analizado (1999-2010). Lógicamente, las grandes empresas realizan o acometen principalmente este tipo de proyectos, mientras que las medianas y pequeñas tendrán unos ratios inferiores. Otro indicador de esta dimensión era el número de adjudicaciones que había obtenido la empresa en el periodo analizado. $Y$ por último, el ratio del importe total adjudicado por la empresa de manera individual entre el importe de la licitación pública del periodo analizado. 
Tabla 8. Clasificación de los concursos por Importe licitado (Maninvest).

\begin{tabular}{|c|c|}
\hline Rango & Importe de concurso \\
\hline 1 & $<600.000 €$ \\
\hline 2 & $600.000-6.000 .000 €$ \\
\hline 3 & $>6.000 .000 €$ \\
\hline
\end{tabular}

- Indicadores relacionales de la obra pública en España. Con esta dimensión se trataba captar la reputación que tenía la empresa a nivel cooperativo. Es decir, si se trataba de una empresa que se adjudicaba muchos proyectos en cooperación con otras empresas, conformando Uniones Temporales de Empresas, o no era así. Los indicadores utilizados fueron el logaritmo (Fombrun y Shanley, 1990) del importe de adjudicaciones que hubiese obtenido la empresa en colaboración con otras, a través de UTE. Y el segundo indicador empleado fue el número de adjudicaciones que hubiese obtenido la empresa en UTE en el periodo analizado (1999-2010).

- Indicadores atributivos de las empresas. En la última dimensión se trata de analizar la reputación de la empresa a nivel individual sin emplear indicadores de la obra pública. En este sentido, Fombrun y Shanley (1990) desarrollaron una serie de indicadores para medir esta reputación, los cuales han sido empleados en este estudio. Los indicadores son los siguientes. En primer lugar, el tamaño de la empresa, que fue computado como el logaritmo de las ventas (ingresos de explotación) del año 2010. El segundo indicador que plantean estos autores es la rentabilidad económica (ROI) del año correspondiente (2010). Y, por último, el riesgo, que es igual al ratio de la desviación típica de la rentabilidad respecto al promedio o media de la rentabilidad. En este estudio, se empleó el ROI para el periodo comprendido entre el año 1999 y 2010.

Con estas tres dimensiones y los indicadores correspondientes, se llevó a cabo un análisis clúster jerárquico con las 229 empresas de la muestra y, usando las distancias euclídeas, fue posible identificar cuatro grandes grupos de empresas claramente diferenciados. Un primer conjunto de empresas, que se identifica como GRUPO A, recogía a los líderes indiscutibles del sector, que eran concretamente seis: Acciona, Dragados, FCC, Sacyr, OHL y Ferrovial-Agromán. Un segundo grupo, GRUPO B, estaba conformado por grandes empresas constructoras de España, algunas de ellas de carácter regional, pero con procesos de expansión nacional, identificando en este grupo concretamente a once empresas, que eran: Comsa, 
S.A., Copisa, Aldesa Construcciones, Viascon, Isolux, Constructora Hispánica, Ploder, Copcisa, Cavosa, Drace y Grupisa Infraestructuras. El tercer grupo identificado es el GRUPO C, que estaba conformado por un conjunto de veintiséis empresas, que podrían ser catalogadas como importantes empresas medianas tanto de ámbito nacional como regional. El último grupo (D), que está formado por el resto de las empresas que conformaban la muestra, aparecía a una distancia geodésica muy lejana respecto a éstas. Este procedimiento es muy similar al empleado por Gulati (1995b) para medir la interdependencia estratégica de las empresas. Así, este autor segmenta cada industria en distintos nichos organizacionales, de tal manera que los miembros de cada nicho poseen recursos y capacidades similares, lo cual les hace diferenciarse del resto de nichos. Para realizar esta segmentación Gulati (1995b) recurre a un algoritmo de clúster jerárquico y mediante las distancias euclídeas evalúa la similitud en cada uno de los conglomerados.

\section{Bloques relacionales}

En segundo lugar, procedimos a identificar bloque relacionales de nuestra muestra recurriendo al concepto de la equivalencia estructural y al algoritmo Concor. La técnica Concor esta basada en la idea de la equivalencia estructural y ha sido frecuentemente usada en el análisis de redes interorganizativas (Nohria, GarciaPont 1991, Oliver 1988). Nosotros usamos este algoritmo, al igual que han hecho otros investigadores (Walker, Kogut \& Shan 1997), para analizar la estructura de alianzas en nuestra red. CONCOR es un algoritmo de clústeres jerárquicos que separa a las empresas en un número de bloques de empresas las cuales siguen un modelo similar de relaciones hasta que un número especificado de bloques ha sido conformado (Nohria, Garcia-Pont 1991, Oliver 1988).

En aras de facilitar la interpretación de los resultados se decidió analizar los patrones relacionales de los tres principales grupos estratégicos eliminando el grupo de las pequeñas empresas (Grupo 1) que realizan una actividad relacional coyuntural.

La aplicación del algoritmo Concor a nuestra muestra nos permitió identificar 4 bloques relacionales. Cada uno de los bloques sigue unos patrones relacionales distintos que vamos a tratar de analizar a continuación. 


\begin{tabular}{|c|c|c|c|}
\hline \multirow{2}{*}{ Bloques } & \multicolumn{3}{|c|}{ Grupos Estratégicos } \\
\hline & 2 & 3 & 4 \\
\hline Bloque 1 & $\begin{array}{c}\text { SANDO ACSA AZVI BEGAR COPASA } \\
\text { ALCUBA SANJOSE COPROSA SACYR } \\
\text { TECSA }\end{array}$ & $\begin{array}{l}\text { CAVOSA DRACE COMSA } \\
\text { COPCISA COPISA ALDESA } \\
\text { GEA ISOLUX ASSIG } \\
\text { FERROSE }\end{array}$ & SACYR \\
\hline Bloque 2 & $\begin{array}{c}\text { TECONSA VIASCON JOCA SORIG } \\
\text { OTIZ PLODE RUBAU RUBAUTA } \\
\text { INTERSA LUBASA PROBISA ASCAN } \\
\text { PAVASAL ELSAN }\end{array}$ & & \\
\hline Bloque 3 & & & $\begin{array}{c}\text { DRAGADOS } \\
\text { FCC }\end{array}$ \\
\hline Bloque 4 & & & $\begin{array}{c}\text { FERROVIAL } \\
\text { ACCIONA } \\
\text { OHL }\end{array}$ \\
\hline
\end{tabular}

La matriz de densidades (Tabla 9) muestra las densidades de las relaciones dentro y entre los distintos bloques.

Tabla 9. Matriz de densidades entre los bloques relacionales.

\begin{tabular}{|l|l|l|l|l|}
\hline & 1 & 2 & 3 & 4 \\
\hline 1 & 0.321 & 0.129 & 0.625 & 0.583 \\
\hline 2 & 0.129 & 0.154 & 0.857 & 0.190 \\
\hline 3 & 0.625 & 0.857 & 1.000 & 1.000 \\
\hline 4 & 0.583 & 0.190 & 1.000 & 1.000 \\
\hline
\end{tabular}

A partir de esta matriz podemos llegar a las siguientes conclusiones: las empresas del bloque 3, Dragados y FCC, constituyen el núcleo de la red, abarcando el 15\% de la actividad relacional que se da en la misma. Este grupo se relaciona principalmente con las empresas "Top" españolas. También presenta una importante correlación a nivel relacional con las empresas que conforman el grupo 2. El grupo 4 que esta conformado por el resto de empresas "Top" se relaciona además de con las "Top" del grupo 3, con las empresas que conforman el grupo 1, que son las grandes de ámbito nacional y Sacyr que también podemos catalogarla 
de "Top". Lo más interesante del grupo 2, empresas medianas, es que sus patrones relacionales convergen con los del núcleo de la red (Dragados y FCC). Por su parte, el grupo 1 conformado por las grandes empresas, y las medianas más importante se correlacionan de manera importante con el grupo 3 y 4 de las empresas "Top" y, por el contrario, con las del grupo 2 mantiene una escasa correlación.

\section{Relación entre bloques relacionales y grupos estratégicos}

Para finalizar, con el objetivo de analizar en qué medida estos bloques relacionales están relacionados con los grupos estratégicos se ha construidos la siguiente tabla. El bloque 3 y 4 están conformados por las empresas más importantes del sector de la construcción. El $82 \%$ de las empresas grandes se ubican en el primer bloque relacional. Por último, las empresas medianas están repartidas entre los dos primeros bloques.

Tabla 10. Relación entre Grupos estratégicos y Bloques relacionales.

\begin{tabular}{|c|c|c|c|}
\hline Bloques relacionales & \multicolumn{3}{|c|}{ Grupos Estratégicos } \\
\hline & 2 & 3 & 4 \\
\hline 1 & $50 \%$ & $45 \%$ & $5 \%$ \\
& $(45,5 \%$ G2) & $(82 \%$ G3) & $(16 \%$ G4) \\
\hline 2 & $85,72 \%$ & $14,28 \%$ & $(18,2 \%$ G3) \\
\hline 3 & $(54,5 \%$ G2) & & $100 \%$ \\
$(33,3 \%$ G4) \\
\hline 4 & & & $100 \%$ \\
& & & $(50 \%$ G4) \\
\hline
\end{tabular}

*No se ha incluido el grupo estratégico 1, ya que este por su escasa actividad relacional fue eliminado para elaborar los bloques relacionales.

\section{Conclusiones}

El presente estudio ha intentado abordar una cuestión ampliamente analizada teóricamente pero escasamente tratada desde un punto de vista empírico, como es la relativa a la estructura relacional de las carteras de alianzas de las empresas. Desde una perspectiva de redes, la cartera o portfolio de alianzas está constituida 
por todos los vínculos directos de cooperación que una empresa mantiene con sus socios. Esta investigación ha explorado las redes ego de las empresas definidas en función de esas alianzas en un contexto de investigación apropiado, tanto por la homogeneidad de las empresas como del tipo de alianzas considerado: las UTES desarrolladas por las empresas de construcción españolas para el desarrollo de las obras públicas. La aplicación del ARS ha permitido caracterizar y analizar las semejanzas y diferencias de las carteras de alianzas de las empresas. El trabajo realizado tiene un carácter exploratorio dada la ausencia de estudios empíricos sobre este tema y ha tenido como finalidad caracterizar esas redes egos que conforman los portfolios de alianzas de las empresas.

Los resultados han puesto de manifiesto diferentes comportamientos estratégicos de las empresas a la hora de diseñar y desarrollar sus carteras de alianzas. Desde un punto de vista relacional se observan notables diferencias en las carteras de alianzas, diferencias que están relacionadas principalmente con el tamaño de las empresas. No obstante, incluso entre las empresas de similar tamaño también se observan divergencias en sus carteras, indicando que las compañías utilizan las alianzas de forma distinta a la hora de desplegar sus estrategias. En todo caso, las mayores diferencias se observan entre grupos de empresas de diferentes tamaños y estrategias. Así, se observa una cierta vinculación, aunque no estricta, entre grupos estratégicos y bloques relacionales, es decir, empresas que pueden ser incluidas dentro del mismo conjunto por tener una cierta equivalencia estructural. Del mismo modo también se aprecian determinados patrones de comportamiento de alianza entre las empresas de los diferentes grupos; en algunos grupos las empresas mantienen fuerte vínculos entre ellas, mientras que en otros las empresas se centran en alianzas con las compañías de otros grupos.

Al tratarse de un estudio exploratorio, de carácter básicamente descriptivo, las limitaciones de la investigación son numerosas, pero ha de entenderse como un primer paso en un proyecto más amplio en el que se pretende estudiar en profundidad los tres aspectos señalados por Wassmer (2010) en el análisis de la cartera de alianzas.

\section{Agradecimientos}

Los autores quieren agradecer las valiosas sugerencias realizadas por el profesor Steve Borgatti en el 1er workshop en investigación de análisis de redes sociales (Ponferrada, 2011). 
Esta investigación ha sido financiada por el Ministerio de Ciencia e Innovación, España (ECO2009-12742).

\section{Referencias bibliográficas}

Ahuja, Gautam (2000a), "Collaboration networks, structural holes, and innovation: A longitudinal study", Administrative Science Quarterly, vol. 45, no. 3, pp. 425.

Baum, Joel A. C.; Calabrese, T. ; Silverman, B.S. (2000), "Don't go it alone: Alliance network composition and startups' performance in Canadian biotechnology", Strategic Management Journal, vol. 21, no. 3, pp. 267.

Borgatti, Stephen P. (1997), "Structural holes: Unpacking Burt's Redundancy Measures.", Connections, vol. 20, no. 1, pp. 35-38.

Borgatti, Stephen P.; Everett, M. ; Freeman, L.C. (2002), Ucinet 6 for Windows: Software for Social Network Analysis. Analytic Technologies: Natick.

Burt, Ronald S. (1992), Structural holes: The social structure of competition, Harvard University Press., Cambridge, MA.

Eccles, Robert G. (1981), "The quasifirm in the construction industry", Journal of Economic Behavior \& Organization, vol. 2, no. 4, pp. 335-357.

Freeman, Linton C. (1978), "Centrality in social networks conceptual clarification", Social Networks, vol. 1, no. 3, pp. 215-239.

Hanneman, R. A. and Riddle, M. (2005), "Introduction to social network methods.", Riverside, CA: University of California, Riverside (published in digital form at http://faculty.ucr.edu/hanneman/.), .

Hoffmann, Werner H. (2007), "Strategies for managing a portfolio of alliances", Strategic Management Journal, vol. 28, no. 8, pp. 827.

Koka, Balaji R. ; Prescott, J.E. (2008), "Designing alliance networks: the influence of network position, environmental change, and strategy on firm performance", Strategic Management Journal, vol. 29, no. 6, pp. 639. 
Koka, Balaji R. ; Prescott, J.E. (2002), "Strategic alliances as social capital: A multidimensional view", Strategic Management Journal, vol. 23, no. 9, pp. 795.

Ministerio de Economía de España (2003), Informe sobre el sector español de obra pública.

Nohria, Nitin ; Garcia-Pont, C. (1991), "Global Strategic Linkages and Industry Structure", Strategic Management Journal, vol. 12, pp. 105.

Oliver, Christine (1988), "The Collective Strategy Framework: An Application To Compet", Administrative Science Quarterly, vol. 33, no. 4, pp. 543.

Ozcan, Pinar ; Eisenhardt, K.M. (2009), "Origin of Alliance Portfolios: Entrepreneurs, Network Strategies, and Firm Performance", Academy of Management Journal, vol. 52, no. 2, pp. 246.

Sarkar, M. B.; Aulakh, P.H. ; Madhok, A. (2009), "Process capabilities and value generation in alliance portfolios", Organization Science, vol. 20, no. 3, pp. 583600.

SEOPAN (2006), Informe anual de la construcción.

Walker, Gordon; Kogut, B. ; Shan, W. (1997), "Social capital, structural holes and the formation of an industry network", Organization Science, vol. 8, no. 2, pp. 109.

Wasserman, Stanley ; Faust, K. (1994), Social Network Analysis. Methods and applications. Cambridge University Press., Cambridge (R.U.). 